\section{(6) OPEN ACCESS}

\title{
Development of patient-centred standards of care for rheumatoid arthritis in Europe: the eumusc.net project
}

\author{
Michaela A Stoffer, ${ }^{1}$ Josef S Smolen, ${ }^{1}$ Anthony Woolf, ${ }^{2}$ Ales Ambrozic, ${ }^{3}$ \\ Ailsa Bosworth, ${ }^{4}$ Loreto Carmona, ${ }^{5}$ Veronika Fialka-Moser, ${ }^{6}$ Estibaliz Loza, ${ }^{5}$ \\ Pawel Olejnik, ${ }^{7}$ Ingemar F Petersson, ${ }^{8}$ Till Uhlig, ${ }^{9}$ Tanja A Stamm, ${ }^{1}$ \\ the eumusc.net-working group
}

\begin{abstract}
Handling editor Hans WJ Bijlsma

- Additional material is published online only. To view please visit the journal online (http://dx.doi.org/10.1136/ annrheumdis-2013-203743).

For numbered affiliations see end of article.
\end{abstract}

\section{Correspondence to}

Dr Tanja Stamm, Department of Internal Medicine III, Division of Rheumatology, Medical University of Vienna, Währinger Gürtel 18-20, Vienna A - 1090, Austria; tanja.stamm@meduniwien.ac. at

Received 8 April 2013 Revised 8 July 2013 Accepted 14 July 2013 Published Online First 6 August 2013

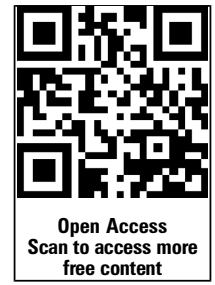

\section{SLinked}

- http://dx.doi.org/10.1136/ annrheumdis-2013-203765

To cite: Stoffer MA, Smolen JS, Woolf A, et al. Ann Rheum Dis 2014;73:902-905.

\section{ABSTRACT}

Objective The eumusc.net project is a European Union (EU) commission and European League Against Rheumatism (EULAR)funded project that aims to facilitate equal standards for musculoskeletal health in all EU countries. One work-package was to develop evidence-based and patient-centred standards of care (SOC), for rheumatoid arthritis (RA) understandable for patients and professionals across Europe.

Method A review of documents covering clinical practice 'guidelines' and SOC for RA was conducted. The obtained documents were evaluated using the Appraisal of Guidelines for Research and Evaluation II (AGREE II) criteria, and all recommended methods to treat RA were extracted. Based on this information, a three-round Delphi exercise was performed including a consensus group meeting of 21 researchers and patient representatives.

Results 16 patient-centred SOC were formulated including a lay version in the format of a checklist. An example is SOC 3: 'People with RA should receive a treatment plan developed individually between them and their clinician at each visit.' The corresponding checklist question reads: 'Have I received a treatment plan which includes an explanation of my management, expected goals and outcomes and important contact details?' Conclusions The SOC for RA will be available in all 23 official European languages and contribute to more unified treatment approaches in Europe.

\section{INTRODUCTION}

Rheumatoid Arthritis (RA) is a chronic inflammatory disease of unknown aetiology characterised by destructive synovitis ${ }^{1}$, with a prevalence of $0.3-1 \%$, and affecting women more frequently than men. ${ }^{2}$ The prevalence varies among countries and regions of the world and appears to be higher in developed countries. $^{2}$ The impact of RA on daily functioning, quality of life $^{4}$ and on employment ${ }^{5}$ is immense. The main target of treatment is to control disease activity. ${ }^{6}$

In attempts to standardise the treatment of RA, clinical practice guidelines have been established in many countries ${ }^{7-19}$ which recommend pharmacological and non-pharmacological treatment, lifestyle advice and patient education. However, guidelines for RA differ considerably between countries.
It is unclear to which extent patients are informed about guidelines even though these primarily relate to their care. The work package 5 of the European Musculoskeletal Conditions Surveillance and Information Network (eumusc.net) assessed pertinent data and focused on the development of a set of recommendations for European Standards of Care (SOC) for people with RA based on existing guidelines. SOC define what services are expedient for people living with a certain condition. They describe management of a disease, care, access to patient information, support and knowledge that patients should have. Furthermore, they focus on structure (eg, availability of health professionals), process (eg, 3-monthly clinical assessments) and outcome (eg, assessment of disease activity). SOC are not guidelines or algorithms of care even if they refer to them ${ }^{20}$; rather, they should be available to patients so they can become empowered to gain responsibility for their disease.

The present study analysed and compared guidelines for RA and, by means of Delphi technique, developed a patient-centred SOC acceptable across all European countries.

\section{METHOD}

A literature search including critical appraisal of national and international guidelines was conducted: subsequently, a consensus meeting followed to achieve expert consensus, employing a Delphi exercise.

\section{Review of literature, critical appraisal and content analysis of RA guidelines}

A systematic review was conducted in Pubmed, CINAHL and the internet (Google) between May 2010 and February 2011 with the following keywords including combinations thereof: clinical practice guideline, guideline, guidance, recommendation, standard of care, rheumatoid arthritis, and all names of European countries. Furthermore, the reference lists of the retrieved documents were reviewed and guideline registers were searched.

Additionally, national societies, health professional associations and patient leagues in the field of rheumatology in 44 European countries were contacted via email and were asked to provide potentially relevant, unpublished documents (see online 


\section{Table 1 Standards of Care (SOC)}

SOC for people with rheumatoid arthritis (RA)

Level of

agreement

SOC 1 People with symptoms of RA should have timely access to a clinician/health professional competent in making a (differential) diagnosis (6 weeks according to European League Against Rheumatism (EULAR) recommendations).

SOC 2 People with RA should be given relevant information and education about

their disease

its management

- and all aspects of living with and managing their RA, in written form and in a format suited and tailored to the individual, in a timely fashion appropriate to their needs.

SOC 3 People with RA should receive a treatment plan developed individually between them and their clinician at each visit.

SOC 4 At the start of any disease-specific treatment, people with RA should be fully educated about the expected benefits and any potential risks, and fully evaluated to assess both clinical status and safety aspects.

SOC 5 People with RA should be fully assessed for symptoms, disease activity, damage, comorbidity (including assessment for cardiovascular disease risk factors) and function at diagnosis; these assessments should also be done annually; if disease is not within target, clinical assessment should be done at least 3-monthly (all clinical variables) and possibly more frequently upon significant worsening.

SOC 6 People with RA should have rapid access to care when they experience significant worsening of the disease.

SOC 7 People with RA should be treated with a disease modifying anti-rheumatic drug (DMARD) as soon as the diagnosis is made.

SOC 8 If the target of low disease activity or remission is not achieved using a synthetic DMARD (usually being methotrexate), treatment should be revaluated at least every 3 months.

SOC 9 People with RA should be evaluated for pain, and relief of pain associated with RA should be considered.

SOC People with RA who have residual joint problems despite state-of-art pharmacological (including intra-articular) and non-pharmacological

10 therapy should be assessed by an orthopaedic surgeon within 3 months from recognition of the refractoriness of the problem, especially if there is joint damage/soft tissue problems that might likely be solved by protective or reconstructive surgery.

SOC People with RA should have access to evidence-based pharmacological and non- pharmacological treatment.

SOC People with RA should have access to a specialised health professional to receive assessment, advice and training in all matters related to

12 their disease.

SOC People with RA should understand the benefit of exercises and physical activity and should be advised to exercise appropriately.

SOC People with RA should receive information, advice and training on joint protection and ergonomic principles as well as activity-based

14 methods to enhance functioning in daily life and participation in social roles. They should receive information, advice and training on splints, aids, devices and other products for environmental adaptations.

SOC People with RA should receive information and advice about

15 a healthy lifestyle (such as discontinuation of all types of tobacco use, balanced use of alcohol, physical activity, healthy diet, management of sleep disturbance if necessary)

- prevention of accidents and injuries,

- support groups and patient organisations,

- when to think about surgery and

- additional treatment options provided that some people might find useful.

SOC People with RA who wish to try alternative therapies that some people found symptomatically beneficial, should be informed about the

16 limited evidence.

These SOC should be a minimum standard for all European countries and should be implemented as soon as possible, but not later than by 2020 .

of the 37 SOC, 21 were excluded because 3 or more experts rated them as not necessary in the third Delphi round. This led to the 16 SOC in the final version. 23 ( $89 \%$ ) participants scored the level of agreement (8.7-9.9).

supplementary table S1). Inclusion criteria for guidelines were as follows: released after January 2002, dealing with the treatment of RA, denoting themselves as a guideline or recommendation with a full text (translation) available in English or German. If more than one version existed, the latest one was included.

The obtained guidelines were appraised based on the Appraisal of Guidelines for Research and Evaluation (AGREE II) instrument to assess their methodological quality. ${ }^{21}$ In order to focus on guidelines with high quality, only documents that received a score between four and seven in the overall assessment were included in the present analysis (see online supplementary figure S1). In the next step, all mentioned interventions for the treatment of RA were extracted. These interventions were linked to the other specifications given in the guidelines, for example, time, intensity and frequency. Based on this information, recommendations were formulated in the form of short sentences.

\section{Delphi exercise}

In the first Delphi round, the interventions were prioritised, clustered and a wording was proposed by a group of 21 experts including four patient research partners. Experts were rheumatologists, rheumatology health professionals and rehabilitation specialists with long-term experience in clinical and/or scientific work.

After a one-day, face-to-face, meeting in Vienna, a set of recommendations was drafted and distributed via email, to all participants for comments. The comments received were built into the second draft, which was again circulated electronically; in the second Delphi round, the participants were asked to agree or disagree with the proposed items. In case of disagreement, a suggestion of an alternative wording should be proposed. Based on this input, a third draft was circulated, the third Delphi round, in which the experts received a feedback about the revisions of the SOC. Following the approach of Freel et $a l^{22}$ the participants were asked to score each SOC using a scale of $1-3$ as follows: $1=\mathrm{A}$ 'must have' for the final set of SOC; $2=$ "Would be nice to have in the final set of SOC'; $3=$ 'Is not important'. In a final voting, the level of agreement was obtained by asking each panel member to score each SOC on a scale from 0 to 10,0 indicated 'I totally disagree' and 10 'I agree completely'. 


\section{RESULTS}

The review of literature yielded 91.247 citations: (Pubmed $\mathrm{n}=89.324$, CINAHL $\mathrm{n}=1.759$, the first 150 Google hits and 14 hits in guideline registers). The titles and/or abstracts were screened and duplicates excluded; 95 documents were obtained in their full version; 87 national scientific societies, social leagues and health professional associations in 44 countries were asked to provide relevant documents. We received 104 documents with a response rate of $63 \%$ to our request (after three personal reminder emails were sent if no response had been received); 14 of these $e^{6-19}$ fulfilled the inclusion criteria and were used for further analysis. Out of all included documents, 49 interventions were extracted. For the Delphi exercise, 24 institutions were informed about the study. Thereof, 15 agreed to participate. Each institution nominated up to two experts for the working group. A total of 26 panel members from 10 countries (as listed in online supplementary appendix A) participated in this activity, including four patient research partners.

\section{Results Delphi round 1}

Twenty-one experts participated in the face-to-face meeting (Vienna 22-02-2012). During the meeting, the 49 interventions were sorted into the following six groups: 1) pharmacological treatment, 2) monitoring, 3) lifestyle interventions, 4) surgery, 5) education/information and 6) non-pharmacological treatment. This resulted in a draft version of 56 proposed items.

\section{Results round 2}

In the second round, 21 (81\%) experts provided 250 comments on the draft version of 56 SOC. The first author modified and reorganised the individual bullet points according to the comments. This process reduced the number of statements to 37 .

\section{Results round 3}

In the third round, 23 (89\%) experts contributed a total number of 202 comments and $21(81 \%)$ rated the importance of each SOC.

Based on the final 16 SOC (table 1), a corresponding checklist worded in a way that could be understood by lay people was formulated (table 2).

\section{DISCUSSION}

To our knowledge, this is the first study developing common European SOC for RA, focusing on a lay version in the format of a checklist. A well-known barrier to the widespread implementation of guidelines and standards in healthcare is the scarcity of high-quality documents in languages other than English. ${ }^{23}$ Providing translations of evidence-based information in all European languages could be a low-cost intervention with potential remarkable impact. Therefore, our document is translated into all European Union (EU) languages to facilitate implementation throughout Europe.

One important aim of the project is to provide tools to reduce inequalities in Europe through spreading evidence-based information in a way that could be understood by lay people as patients usually are.

The greatest challenge in this work on European SOC was to agree on the wording that was most appropriate and applicable for all European countries. Among these challenges is the fact that therapeutic interventions in RA are carried out by different

\section{Table 2 Checklist}

\section{What this means for you and your rheumatoid arthritis (RA)...}

1 Was my RA diagnosed by a specialised health professional within 6 weeks of onset of symptoms?

2 Do I understand my disease, my role in its management and the role of health professionals? Have I been given information in different formats and/or education about my disease? Have I been given information and/or education about treatments, their benefits and risks? Have I been given information and education relevant to my needs, that is, what to do if my disease is worsening, in case of pain and adverse reactions on drugs? Have I been given information about, and given contact details of, relevant patient charities and organisations which are considered to be trusted sources of evidence-based information?

3 Have I received a treatment plan which includes an explanation of my management, expected goals and outcomes and important contact details?

4 Was I informed about expected benefits and potential risks of treatment? Was I assessed for clinical status and safety before the treatment was started? Was I informed about vaccinations?

5 Have I received a schedule of regular assessments of my disease - the symptoms, disease activity and of what I can do?

6 Have I been informed when, how and who I can contact in case my disease is worsening?

7 Am I receiving a disease modifying anti-rheumatic drug, and if not, do I understand why not?

8 If my target of low disease activity or remission is not achieved, is my treatment reappraised at least every 3 months?

9 Do I know how to control pain associated with my RA?

10 Have I been informed about the options of surgery, and have the benefits and risks been explained?

11 Do I have access to pharmacological and non-pharmacological treatments according to my needs?

12 Do I have the opportunity to receive support if needed from health professionals, such as rheumatologist, dietician, general practitioner, nurse, occupational therapist, physiotherapist, psychologist and social worker?

Have I been offered information about how, why and when to contact different members of the multidisciplinary team as soon as possible after my diagnosis was made?

13 Have I been informed about physical activity and exercises specific for me?

14 Have I received information and, if necessary, advice and training on aids, devices and ergonomic principles to enhance function in daily life and participation in social roles?

15 Have I been informed about a healthy lifestyle?

16 Have I been informed about alternative therapies and the current (limited) evidence available to make an informed treatment decision?

This checklist was developed by patient research partners in collaboration with the whole working group. The patient research partners have been recruited through patient leagues participating in this work package.

We suggest this checklist should be available for all patients, throughout Europe but should particularly be a source for active and interested patients.

The checklist should enable patients in Europe to follow the adherence to the recommended standards of care of their RA. 
health professionals in different European countries and, indeed, certain health professionals, for example, specialised rheumatology nurses exist in only a minority of European countries. Another challenge in the course of the formulation of the SOC was finding the appropriate terms that would provide enough information without being too constraining.

Our study has several limitations. First, we restricted the guidelines solicited to those available in English or German versions; second, the number of patient research partners was not very large and third, we did not perform empirical testing to be informed about the applicability of the SOC. Furthermore, we did not re-examine the evidence base of the guidelines. Nevertheless, this activity allowed us to bring the currently available documents together in an attempt at unification. The extent of implementation should be subject to future studies.

Based on the SOC, quality indicators have been developed in another work package. ${ }^{24}$

The standards will be available on the website (http://www. eumusc.net) of the project with the possibility to provide comments. These comments will be considered for a potential update of the document that will be developed if the evidence base has changed substantially and modifications become necessary.

In summary, this work provides a set of evidence-based SOC for the treatment of RA in all languages of the European Union, to be applied by healthcare providers; in particular, these SOC can be understood by patients, and their dissemination will, hopefully, lead to identical treatment approaches across European countries.

\author{
Author affiliations \\ 'Internal Medicine III, Division of Rheumatology, Medizinische Universität Wien, \\ Vienna, Austria \\ ${ }^{2}$ Duke of Cornwall Rheumatology Unit, Royal Cornwall Hospital, Truro Cornwall, UK \\ ${ }^{3}$ University Medical Centre Ljubljana, Ljubljana, Slovenia \\ ${ }^{4}$ National Rheumatoid Arthritis Society, Berkshire, UK \\ ${ }^{5}$ Instituto de Salud Musculoesqueletica, Madrid, Spain \\ ${ }^{6}$ Department for Physical Medicine and Rehabilitation, Medizinische Universität \\ Wien, Vienna, Austria \\ ${ }^{7}$ Instytut Reumatologii, Warsaw, Poland \\ ${ }^{8}$ Orthopedics and Rheumatology, Clinical Sciences, Lund University, Lund, Sweden \\ ${ }^{9}$ National Resource Center for rehabilitation in Rheumatology, Diakonhjemmet \\ Hospital, Oslo, Norway
}

Contributors All persons contributing to this work are listed in online supplementary appendix A, "Expert Panel".

Funding This project was funded by the European Community (EC Community Action in the Field of Health 2008-2013) and by the European League Against Rheumatism (EULAR) (grant number 20081301EU). The views of the funding body did not influence the content of the work.

Competing interests AnB has received research grants from AbbVie, Merck, Amgen and an educational grant from Pfizer; none of them relates to this work. IFP has received speaker fees from AbbVie, Pfizer and UCB. JS has received honoraria from AbbVie, Amgen, BMS, Wyeth, Jansen, Roche, Schering-Plough and Sanofi-Aventis. TS has received speaker fees from UCB, AbbVie and MSD. MS has received speaker fees from MSD.

Provenance and peer review Not commissioned; externally peer reviewed.

Open Access This is an Open Access article distributed in accordance with the Creative Commons Attribution Non Commercial (CC BY-NC 3.0) license, which permits others to distribute, remix, adapt, build upon this work non-commercially, and license their derivative works on different terms, provided the original work is properly cited and the use is non-commercial. See: http://creativecommons.org/ licenses/by-nc/3.0/

\section{REFERENCES}

1 Helmick CG, Felson DT, Lawrence RC, et al. Estimates of the prevalence of arthritis and other rheumatic conditions in the United States. Arthritis Rheum 2008;58:15-25.

2 World Health organization (WHO). World report on disability 2011. 2011; Retrieved 1 Jun 2012. http://whqlibdoc.who.int/publications/2011/9789240685215 eng.pdf

3 Alamanos Y, Voulgari PV, Drosos AA. Incidence and prevalence of rheumatoid arthritis, based on the 1987 American College of Rheumatology criteria: a systematic review. Semin Arthritis Rheum 2006;36:182-8.

4 Loza $E$, Jover JA, Rodriguez $L$, et al. Multimorbidity: prevalence, effect on quality of life and daily functioning, and variation of this effect when one condition is a rheumatic disease. Semin Arthritis Rheum 2009:38:312-9.

5 Franke LC, Ament AJ, van de Laar MA, et al. Cost-of-illness of rheumatoid arthritis and ankylosing spondylitis. Clin Exp Rheumatol 2009;27:118-23.

6 Smolen JS, Aletaha D, Bijlsma JW, et al. Treating rheumatoid arthritis to target: recommendations of an international task force. Ann of the Rheum Dis 2010;69:631-7.

7 Haute Autorite de Sante (HAS). Rheumatoid arthritis, Treatment of established RA 2007; Retrieved 2 Jan 2012. http://www.has-sante.fr/portail/upload/docs/ application/pdf/pr_prise_en_charge_en_phase_detat_recommandations.pdf

8 Gossec L, Pavy S, Pham T, et al. Nonpharmacological treatments in early rheumatoid arthritis: clinical practice guidelines based on published evidence and expert opinion. Joint, Bone, Spine. Revue Du Rhumatisme 2006;73:396-402.

9 Forestier R, André-Vert J, Guillez P, et al. Non-Drug treatment (excluding surgery) in rheumatoid arthritis: clinical practice guidelines. Joint, Bone, Spine. Revue Du Rhumatisme 2009:76:691-8.

10 Fautrel B, Pham T, Gossec L, et al. Role and modalities of information and education in the management of patients with rheumatoid arthritis: development of recommendations for clinical practice based on published evidence and expert opinion. Joint, Bone, Spine. Revue Du Rhumatisme 2005;72:163-70.

11 Haute Autorite de Sante (HAS). Rheumatoid arthritis, Medical social and organisational aspects of the treatment (excluding surgery anddrugs). 2007; Retrieved 2 Jan 2012. http://www.has-sante.fr/portail/upload/docs/application/pdf/ rheumatoid arthritis guidelines.pdf

12 Gossec L, Fautrel B, Pham T, et al. Structural evaluation in the management of patients with rheumatoid arthritis: development of recommendations for clinical practice based on published evidence and expert opinion. Joint, Bone, Spine. Revue Du Rhumatisme 2005;72:229-34.

13 Schneider $M$, Gasser $A$. Interdisziplinäre leitlinie management der frühen rheumatoiden arthritis. Steinkopff Verlag Darmstadt 2007. http://www.bdrh.de/ fileadmin/redaktion/Leitlinie fruehe RA.pdf

14 Scottish Intercollegiate Guideline Network (SIGN). Management of early rheumatoid arthritis. 2007; Retrieved 3 Jan 2012. http://www.sign.ac.uk/pdf/sign123.pdf

15 Spanish Society of Rheumatology (SER). Update of the clinical practice guideline for the management of rheumatoid arthritis in Spain. 2007; Retrieved 3 Jan 2012 http://www.ser.es/ArchivosDESCARGABLES/Proyectos/GUIPCAR_2007/ GUIPCAR2007-ENG.pdf

16 Luqmani R, Hennell S, Estrach C, et al. British society for rheumatology and British health professionals in rheumatology guideline for the management of rheumatoid arthritis (after the first 2 years). Rheumatology (Oxford) 2009:48:436-9.

17 Luqmani R, Hennell S, Estrach C, et al. British society for rheumatology and British health professionals in rheumatology guideline for the management of rheumatoid arthritis (the first two years). Rheumatology (Oxford) 2006;45:1167-9.

18 Kennedy T, McCabe C, Struthers G, et al. BSR guidelines on standards of care for persons with rheumatoid arthritis. Rheumatology (Oxford) 2005:44:553-6.

19 National Collaborating Centre for Chronic Conditions. Rheumatoid arthritis: national clinical guideline for management and treatment in adults. London: Royal College of Physicians, 2009; Retrieved 3 Jan 2012. http://www.nice.org.uk/nicemedia/live/ 12131/43326/43326.pdf

20 Arthritis and Musculoskeletal Alliance (ARMA). Standards of Care for people with Inflammatory Arthritis. 2004; Retrieved 1 Jun 2012. http://www.nras.org.uk/ includes/documents/cm_docs/2012/a/arma_standards_of_care_for_ia.pdf

21 Appraisal of Guidelines for Research and Evaluation Trust (AGREE) Next Steps. The AGREE II Instrument (Electronic version). 2009; Retrieved 1 June 2012. http:www. agreetrust.org

22 Freel $A C$, Shiloach $M$, Weigelt JA, et al. American college of surgeons guidelines program: a process for using existing guidelines to generate best practice recommendations for central venous access. J Am CollSurg 2008;207:676-82.

23 Henrotin $Y$. Need for high-standard translation methodology for the dissemination of guidelines. Osteoarthritis Cartilage 2009;17:1536-8.

24 Petersson IF, Strömbeck B, Andersen L, et al. Development of Health Care Quality Indicators for Rheumatoid Arthritis in Europe: the eumusc.net project. Ann Rheum Dis 2014;73:902-5. 